\title{
El Jornal das Senhoras: un proyecto periodístico femenino para la emancipación de las mujeres brasileñas
}

\author{
Jornal das Senhoras: a female journalistic project for the emancipation of Brazilian \\ women \\ Regina Simon da SILVA* \\ Universidade Federal do Rio Grande do Norte (UFRN)
}

\begin{abstract}
RESUMEN: La argentina Juana Paula Manso (1819-1875) nació y vivió la mayor parte de su vida en uno de los períodos más conturbados de la historia de Argentina, y sus ideales, osados para la mentalidad de la época, le trajeron, entre tantos problemas, persecuciones políticas por parte de Juan Manuel de Rosas, que culminaron en el exilio de la familia, primero en Uruguay y luego en Brasil. En este país, Manso fundó y dirigió, en 1852, el Jornal das Senhoras, considerado el primer periódico feminista brasileño, con la clara intención de promover la emancipación de las mujeres. El presente artículo, dedicado a la prensa femenina, analiza el diálogo establecido entre el Jornal das Senhoras y las lectoras brasileñas, buscando identificar elementos que fomenten su ilustración y emancipación.
\end{abstract}

PALABRAS CLAVES: Juana Manso. Prensa feminina. Jornal das Senhoras. Emancipación.

\begin{abstract}
Argentina's Juana Paula Manso (1819-1875) was born and lived most of her life in one of the most troubled periods in the history of Argentina, and her ideals, daring for the mentality of the time, brought her, among many problems, and political persecutions by Juan Manuel de Rosas, which culminated in the family's exile, first to Uruguay and then to Brazil. In this country, Manso founded and directed, in 1852, Jornal das Senhoras (Women Journal), considered the first Brazilian feminist newspaper, with the clear intention of promoting the emancipation of women. This article, dedicated to the female press, analyzes the dialogue established between the Jornal das Senhoras and the Brazilian readers to identify elements that promote their illustration and emancipation.
\end{abstract}

KEYWORDS: Juana Manso. Women Press. Jornal das Senhoras. Emancipation.

\footnotetext{
* Professora Associada de Língua e Literatura Hispânicas no Departamento de Línguas e Literaturas Estrangeiras Modernas da Universidade Federal do Rio Grande do Norte - UFRN e do Programa de Pós graduação em Estudos da Linguagem - PpgEL. Pós-doutorado em Letras Neolatinas da Universidade Federal do Rio de Janeiro - UFRJ (2019), opção Literatura Hispano-americana e Doutorado e Mestrado pela mesma instituição. E-mail: reginasimonsilva@gmail.com.
} 


\title{
Unas pocas palabras sobre Juana Paula Manso
}

\author{
¿A Minerva te consagras? \\ Perdone Amor tu imprudencia: \\ Advierte que tanta ciencia \\ No es propia de la beldad. \\ No: tu sencillez conserva, \\ Y esa feliz ignorancia \\ Que la deliciosa infancia \\ Te recuerdan sin cesar.
}

(José María Heredia)

Quisiera empezar mi estudio con este epígrafe porque estos versos ilustran románticamente el pensamiento masculino con relación a las mujeres a comienzos del siglo XIX en América Latina. El paralelismo que se establece entre las imágenes "feliz ignorancia" y "deliciosa infancia" es una estrategia para embellecer lo feo, es decir, se utiliza la inocencia de la infancia para persuadir a la mujer de lo bueno que es permanecer lejos de la ciencia. Para Mary Louise Pratt (1993, p. 54), ese poema, "leído en el contexto de las luchas alrededor de género y ciudadanía en las Américas recientemente independientes, presenta una verdadera prescripción para la subordinación femenina".

En efecto, la independencia de las colonias españolas en América no produjo cambios significativos para las mujeres. Las disputas para llegar al poder privilegiaban las conquistas masculinas, mientras que las mujeres blancas, burguesas, permanecían restringidas a los espacios privados del hogar, educadas para mantener la felicidad hogareña, independiente de su voluntad. Sin embargo, este estereotipo femenino sostenido por el patriarcado no se aplicaba a todas las mujeres en diferentes épocas, pues como afirma Lerner:

\footnotetext{
Siempre ha existido una pequeña minoría de mujeres privilegiadas, por lo general pertenecientes a la elite dirigente, que han tenido acceso al mismo tipo de educación de sus hermanos. De entre sus filas han salido las intelectuales, las pensadoras, las escritoras, las artistas (...). (LERNER, 1990, p. 12).
}

Entre ese pequeño grupo de mujeres ilustradas del siglo XIX en América Latina se encuentra la argentina Juana Paula Manso de Noronha (1819-1875), escritora, 
traductora, poeta, profesora, periodista y precursora del feminismo en Argentina, Uruguay y Brasil. En 1840 Manso vivió la experiencia del exilio cuando su familia se traslada a Montevideo, huyendo de la dictadura de Juan Manuel de Rosas ${ }^{1}$. Al ser sitiada esta ciudad por Manuel Oribe, en 1842, la familia se dirige hacia Brasil. En Rio de Janeiro Manso conoce al violinista portugués Francisco Saá de Noronha, con quien se casa, en 1844. Con su esposo viaja en turné a los Estados Unidos, en 1846, donde nace Eulalia, su primera hija. Allí entabla amistad con Horace Mann, quien le posibilita ampliar sus conocimientos pedagógicos. Luego la familia se dirige a Cuba, donde nace su segunda hija, Herminia.

El vaivén de Manso por diversos países, y el contacto con distintas culturas, intelectuales, personas comunes y experiencias intensas le exigen constantes reajustes ideológicos que se reflejan en su aprendizaje y en su manera de ver el mundo "en una lógica de mestizaje cultural que construye identidades nacionales a partir de la idea de que la soberanía cultural no reside en rechazar lo otro sino incorporarlo" (ANTELO, 2016, p. 212).

Con ese "repertorio cultural" adquirido -y de regreso a Brasil- Manso buscó una forma de transmitir sus ideas y vio en el periodismo una oportunidad. Para ello, fundó y dirigió, en 1852, el Jornal das Senhoras: Modas, Litteratura, Bellas Artes, Theatros e Crítica, objeto de estudio del presente artículo, que pretende analizar el diálogo establecido entre el Jornal das Senhoras y sus lectoras, buscando identificar elementos que fomenten su ilustración y emancipación, y de qué forma eso influenció en el comportamiento y cambio de actitudes de las brasileñas.

\section{El Jornal das Senhoras: datos generales y estructurales}

El Jornal das Senhoras, considerado durante mucho tiempo como el primer periódico brasileño dirigido por mujeres ${ }^{2}$ orientado al público femenino, empezó a circular el 01 de enero de 1852, en Rio de Janeiro, y finalizó sus actividades en

\footnotetext{
${ }^{1}$ Durante el segundo gobierno de Rosas (1835-1852) hubo un éxodo en masa de intelectuales y escritores de Argentina.

${ }^{2}$ Según Zahidé Lupinacci Muzart (2003) el primer periódico fundado por una mujer en Brasil fue Belona Irada contra os Sectarios de Momo, fundado por Maria Josefa Barreto (1786-1837), en Porto Alegre, que circuló entre noviembre de 1833 y enero de 1834. Curiosamente, "Bellona" será un seudónimo utilizado por Manso como recurso al escribir algunos de sus artículos en el Jornal das Senhoras.
}

Revista Moara, n. 56, vol. 1, ago-dez 2020 ISSN: 0104-0944 
diciembre de 1855, un tiempo relativamente largo si consideramos el carácter efímero de esas revistas. Durante los cuatro años de circulación tuvo tres redactoras jefes: 1) Juana Paula Manso de Noronha, de enero a junio de 1852; 2) Violante Atabalipa Ximenes de Bivar e Vellasco, entre julio de 1852 y mayo de 1853 y 3) Gervasia Nunezia Pires dos Santos Neves, entre junio de 1853 y diciembre de 1855 . Y tuvo cinco direcciones tipográficas distintas. Bajo la dirección de Manso fue impreso primeramente en la "Typographia Parisiense", ubicada en la Calle do Ouvidor, n. 20, y a partir de la décima edición (07/03/1852) la impresión estuvo a cargo de la "Typographia de Santos e Silva Junior", en la Calle da Carioca, n. 32. Circulaba los domingos, pero el primer número, con fecha de 1 de enero de 1852, salió un jueves (COSTA, 2012, p. 213).

Compuesto de ocho páginas con dos columnas, utilizó el sistema común de la época, la numeración continuada, empezando un nuevo contaje cuando cambiaba de redactora. Las páginas que corresponden al período de actuación de Manso van de la página 1 hasta la 215 , un total de 26 números.

El programa y funcionamiento del semanario venía en la última página, así como el nombre de las casas (tiendas) donde se realizaban las suscripciones y retiradas de los figurines etc., todas las tiendas en la Calle del Ouvidor. Según Barbosa (s,f) eso demuestra la visión estratégica de la directora, pues, al establecerse en una calle muy concurrida por el público femenino - lo que le daba visibilidad entre los que accedían a estos establecimientos-, los propietarios también se beneficiaban con el nuevo atractivo y la divulgación de sus tiendas.

Su contenido, bastante diversificado, no seguía una programación fija en cuanto al orden de su presentación, lo que le daba un carácter dinámico y muestras que la redactora estaba al día con las últimas noticias de la época. Así, el semanario solía traer: carta de la redactora y/o lectoras; moda -siempre de inspiración parisiense-; artículos en los que Manso divulgaba sus ideas de ilustración y emancipación de las mujeres-; teatro, siempre acompañado de una crítica; poesías; crónica de la semana; música preferentemente una partitura de su esposo Noronha-; bordados y el folletín Misterios del Plata: romance historico contemporaneo, finalizado ( $\sin$ epílogo) en la edición del día 04 de julio de 1852, cuando Manso ya se había retirado de la redacción del periódico, dejando una "nota de la autora" en que habla de su novela: 
Comencé el bosquejo de esta novela en Filadelfia, en 1846; fue concluida en la fortaleza de Garavatá, donde viví cinco meses, en fines de 1849 y principios de 1850. (...) El epílogo de la presente novela no ha podido por el momento publicarse en el Jornal das Senhoras por inconvenientes independientes de nuestra voluntad; pero estamos dispuestas, siempre que tengamos cooperación, a hacer una edición de los Misterios acompañada entonces del epílogo. ${ }^{3}$ (Jornal das Senhoras, Rio de Janeiro, año II, n. 1, 4 de jul. de 1852, p. 8).

\section{Un proyecto de ilustración para la emancipación de la mujer en Brasil}

Si en el Brasil del siglo XIX la lectura en general era escasa, entre las mujeres la situación era aún peor. Pocas eran las lectoras, inclusive entre las familias más ricas, pues el patriarca creía que la "ilustración" era contraria al papel atribuido, tradicionalmente, a las mujeres, como señala las investigadoras Lajolo y Zilberman (2019, p. 329-330):

El universo de lectura de la mujer brasileña es de lo más restringido, lo que, por cierto, sintoniza bastante con la sociedad en que vive. Analfabeta en la mayoría de los casos, la mujer brasileña forma parte de un mundo para el cual el libro, la lectura y la alta cultura no parecen tener mayor significado. ${ }^{4}$

En ese sentido se puede evaluar la tarea doble de Manso, pues para lograr su propósito de emancipación de las mujeres, primeramente había que ultrapasar la barrera de la propia sociedad, que sostenía que "feminidad y saber se excluyen. La lectura abre las puertas peligrosas del imaginario. Una mujer culta no es una mujer"5 (PERROT, 2017, p. 93). Ignorando este pensamiento misógino del cual Manso será víctima

\footnotetext{
${ }^{3}$ En el original: "Comecei a esboçar este romance em Philadelphia, em 1846; foi concluido na fortaleza do Garavatá, onde morei cinco mezes, em fins de 1849 e principios de 1850. (...) O epilogo do presente romance não é possivel por ora publicar-se no Jornal das Senhoras por inconvenientes independentes da nossa vontade; mas estamos disposta, sempre que acharmos cooperação, a fazer uma edição dos Mysterios acompanhada então do epilogo". (Mantuvimos la ortografía del portugués de la época, siglo XIX, en todas las citas del periódico).

${ }^{4}$ En el original: "O universo de leitura da mulher brasileira é dos mais restritos, no que, aliás, se afina bastante à sociedade em que vive. Iletrada na maioria dos casos, a mulher brasileira faz parte de um mundo para o qual o livro, a leitura e a alta cultura não parecem ter maior significado".

${ }^{5}$ En el original: "feminilidade e saber se excluem. A leitura abre as portas perigosas do imaginário. Uma mulher culta não é uma mulher".
} 
posteriormente $^{6}$, la periodista inaugura su semanario con una carta dirigida "A nuestras suscriptoras"7 en la que presenta el objetivo de la redactora que "Si no tiene talento, al menos tiene la voluntad y el deseo de propagar la ilustración y cooperar con todas sus fuerzas para la mejoría social y la emancipación moral de la mujer" ${ }^{8}$ ( $O$ Jornal das Senhoras $^{9}$, Rio de Janeiro, ano I, n. 1, 1 jan. 1852, p. 1).

A continuación, Manso reflexiona acerca del prestigio y visibilidad que la figura del redactor despierta entre el círculo de los intelectuales, que para muchos "hace crecer dos palmos a cualquiera" ${ }^{\prime 10}$ (JS, n. 1, 1852, p. 1); pero también señala que la gente común, que no valora el oficio del intelectual, considera a los literatos como "una casta de vagos (...) un ser inútil" "11 (JS, n. 1, 1852, p. 1). Por si no fueran suficientes esas visiones antagónicas sobre un mismo oficio, Manso imagina un posible cuestionamiento de las lectoras sobre la presencia de una mujer ejerciendo esa función: “¿qué bicho de siete cabezas será?"12 (JS, n. 1, 1852, p. 1). Como respuesta, desmitifica la cuestión argumentando que en muchos países de Europa y en los Estados Unidos esa práctica ya es una realidad. Y para persuadir a las lectoras a formar parte de este movimiento innovador, Manso defiende que América no puede quedar ajena al progreso, haciéndoles un fuerte llamado a la cooperación:

\footnotetext{
Aquí estamos en campaña; el estandarte de la ilustración ondula con gracia en la fragante brisa de los trópicos: bienvenidas a él, todas las que tienen una chispa de inteligencia, vengan. Confidente discreto de sus producciones literarias, serán publicadas bajo anonimato; pero no teman confiárnoslas, ni teman expandir su pensamiento; si lo poseen, es porque es un regalo de la Divinidad, y aquello que Dios da, los hombres no pueden robarlo. ${ }^{13}$ (JS, n. 1, 1852, p. 1)
}

\footnotetext{
${ }^{6}$ Domingo Faustino Sarmiento, que la consideraba amiga y colaboradora, así se expresa sobre su condición de mujer: "La Manso, a quien apenas conocí fue el único hombre en tres o cuatro millones de habitantes en Chile y la Argentina que comprendiese mi obra de educación (...) ¿Era una mujer?” (apud ZUCCOTTI, 1994, p. 102).

${ }^{7}$ En el original: "As nossas Asignantes".

${ }^{8}$ En el original: "senão possue talentos, pelo menos tem a vontade e o desejo de propagar a ilustração e cooperar com todas as suas forças para o melhoramento social e para a emancipação moral da mulher".

${ }^{9}$ En las citaciones siguientes indicaremos el Jornal das Senhoras con las iniciales JS acompañadas del número del periódico, año y página correspondiente.

${ }^{10}$ En el original: "faz crescer dous palmos a qualquer individuo".

${ }^{11}$ En el original: "uma casta de vadios (...) um ente inutil".

${ }^{12}$ En el original: "Que bicho de sete cabeças será?".

13 En el original: "Eis-nos pois em campanha; o estandarte da ilustração ondula gracioso á briza perfumada dos Tropicos: acolhei-vos a elle, todas as que possuis uma faisca de intelligencia, vinde.
} 
Diferentemente de las lectoras/colaboradoras, que tendrán sus identidades protegidas, Manso no teme exponer su nombre, firmando orgullosamente la carta: “Joanna Paula Manso de Noronha”. Según Batticuore (2005, p. 132), este hecho es, sin duda, "un rasgo moderno que la singulariza y la desmarca de la mayoría de sus predecesoras y también de muchas de sus contemporáneas, inaugurando a través de la prensa femenina (...) la modalidad de la autoría apropiada y exhibida" [la bastardilla es de la autora].

Dueña de un discurso crítico, irónico y jocoso, Manso deja muy evidente que los textos divulgados son suyos y se exhibe cuando procura definir lo que es una mujer según su mirada, cuestionándose -y a la vez a las lectoras-, “¿Qué es la mujer?”. Con eso, Manso anticipa en casi un siglo la polémica pregunta hecha por Simone de Beauvoir y divulgada en la primera página de su libro El segundo sexo, en 1949. Manso critica las definiciones misóginas y machistas proferidas por los hombres y que forman parte del imaginario masculino como: "¡la mujer! es un demonio con faldas, una serpiente, un monstruo, una furia, etc."14 (JS, n. 1, 1852, p. 5), concepciones que todavía se mantienen en las sociedades modernas y que niegan a las mujeres sus derechos, perjudicando su porvenir.

Sin una respuesta definitiva a la pregunta, Manso contesta con lo obvio: "la mujer no es el hombre" ${ }^{15}$ (JS, n. 1, 1852, p. 5). Pero, al atribuirles a ellas las mismas características como alma, inteligencia y derechos, las coloca en pie de igualdad con ellos: "La mujer en fin no es, a nuestro entender, un ser aparte de la creación, y entra en el reparto con el hombre - del bien y del mal- de la inteligencia y la estupidez"16 (JS, n. 1, 1852, p. 6). Reivindica una reforma en la educación moral para que los hombres dejen de considerarlas su propiedad, que la mujer "Sea como Dios la hizo: ser que piensa, y no cosa que se cambie de su sitio sin ser consultada"17 (JS, n. 1, 1852, p. 6).

Confidente discreto das vossas producções litterarias, ellas serão publicadas debaixo de anonimo; porem não temaes confiar-mo-las, nem temaes dar expansão ao vosso pensamento; se o possuis é porque é dom da Divindade, e aquillo que Deus dá, os homens não podem roubar”.

${ }^{14}$ En el original: "a Mulher! é um demonio com saias, uma cobra, um monstro, uma furia, etc.".

${ }^{15}$ En el original: "a mulher não é o homem”.

${ }^{16}$ En el original: “A mulher em fim não é em nosso entender um ser á parte na criação, e entra na partilha com o homem - do bem e do mal - da intelligencia e da estupidez".

${ }^{17}$ En el original: "seja o que Deos a fez: ser que pensa, e não coisa que se muda de logar sem ser consultada". 
Finaliza el ensayo diciendo a qué vino, que el Jornal va a tratar de esos derechos y de esa educación, condición necesaria para que las mujeres logren su emancipación.

Pero ¿cómo conquistar la confianza y el interés de las lectoras para convencerlas de abrir las páginas del Jornal? Al analizar este periódico se deduce que Manso demuestra tener conciencia de que es preciso que haya un atractivo para ello, y la moda surge como una salida, una vía muy eficaz para seducir a las lectoras. Este aprendizaje Manso lo asimiló de las experiencias de la prensa francesa, pues, según Perrot (2017, p. 33), las primeras lectoras del siglo XVIII, en Francia, no demostraban interés por los periódicos de contenido político, destinados más a los hombres, pero se convirtieron en fieles lectoras de las columnas de los folletines y de las secciones de moda, lo que llevó a la prensa a especializarse en ese tipo de segmento que tendrá gran desarrollo en el siglo XIX, en razón del éxito entre las mujeres.

María Vicens (2017), en su artículo "Entretenimiento, público y autoría femenina en los periódicos de Juana Manso (1850-1860)", subraya la mirada renovada por parte de estudiosos que han investigado este fenómeno, destacando los trabajos de Regina Root y Susan Hallstead que "han revisitado gran parte de este corpus de periódicos para recortar lo que denominan fashion narratives y pensar cómo la moda dialoga con los discursos políticos de su tiempo y ocupa lugar importante en la construcción de la identidad nacional" (apud VICENS, 2017, p. 51), [la bastardilla es de la autora]. La autora también señala los estudios de Victor Goldgel que ampliaron las discusiones sobre el tema al analizar "el uso de lo frívolo para conquistar lectores" al que se pretende “"civilizar' y 'modernizar”" (apud VICENS, 2017, p. 51).

En vista de ello Manso anuncia, en la última página de la primera edición, el atractivo del próximo número: "Advertimos a nuestras suscriptoras que para el siguiente número daremos un patrón de varios bordados muy hermosos y el molde del -chaleco de emancipación- que todas las señoras deben usar"18 (JS, n. 1, 1852, p. 8). Por detrás de este anuncio banal, se observa, en la elección del nombre de la prenda, "chaleco de emancipación", y en el uso del verbo “deber" -que indica una obligación, un compromiso- la intención de la redactora de influenciar el comportamiento de sus lectoras con relación a lo que ellas deben vestir. Para seducirlas utiliza como estrategia

\footnotetext{
${ }^{18}$ En el original: "Advertimos as nossas assignantes que para o seguinte numero daremos um padrão de diversos bordados mui lindos, e o molde do - colete de emancipação - que todas as senhoras devem uzar".
} 
el interés de las mujeres por estar a la moda. No se trata de una prenda cualquiera, el chaleco es, simbólicamente, un signo de identidad que pretende destacar las lectoras del Jornal das Senhoras de las demás mujeres de la sociedad carioca.

Como prometido, el número siguiente empieza con la sección "Modas" ocupando lugar de relieve. La colaboradora anónima ${ }^{19}$ dedica dos páginas para describir el chaleco de emancipación y su fama entre las parisienses, una moda capaz de "Producir una revolución y un furor que, como la electricidad, tocará todos los rincones de Francia" ${ }^{20}$ (JS, n. 2, 11 enero 1852, p. 9). Con ese argumento la colaboradora procura persuadir a las lectoras de usar una moda que, seguramente, rompe con los patrones dictados a las mujeres por la "buena sociedad", por eso la entonación de sorpresa:

\footnotetext{
Pero un chaleco, un chaleco de hombre (¡vean qué diferencia!), bien cortado, con su cuello en pie, o de solapa, o traspase, empleado en el cuerpo delgado y piramidal de una niña de quince a veinte años, o incluso de una señora hasta los treinta y cinco años, ciertamente es muy hermoso ${ }^{21}$ (JS, n. 2, 1852, p. 10).
}

Según Almeida (1996) y Piscitelli (1998) "La dicotomía femenino y masculino fue y es utilizada como un mecanismo de diferenciación y poder entre las relaciones sociales"22 (apud PERLIN; KISTMANN, 2018, p. 6). Por lo tanto, vemos que, adaptar una prenda típicamente masculina para su uso entre las mujeres es una manera de romper con la supremacía masculina caracterizada por la división binaria hombre/mujer-, una posibilidad viable a partir de la emancipación del sexo femenino, comenzando por la libertad de elegir la ropa que le conviene llevar.

Por supuesto el proyecto de Manso no está pensando solamente en cambios relacionados a la moda, lo que por sí solo ya provoca discusión, sino que, y principalmente, busca una transformación en lo íntimo de las mujeres, pues solo con la ilustración y la educación del sexo femenino es posible desarrollar la inteligencia y,

\footnotetext{
${ }^{19}$ Que puede tratarse de la propia Juana Manso, pero no hay estudios que comprueban eso.

${ }^{20}$ En el original: "produzir uma revolução e um furor que, como a electricidade, vae tocar todos os pontos da França".

${ }^{21}$ En el original: "Mas um colete, um colete de homem (ora vejão que differença!) bem talhado, com sua gollinha em pé, ou de rebuço, ou de traspasse, empregado sobre o corpo esbelto e piramidal de uma menina de quinze a vinte annos, ou mesmo de uma senhora até aos seus trinta e cinco, é por certo mui bonito".

${ }^{22}$ En el original: "a dicotomia feminino e masculino foi e é utilizada como mecanismo de diferenciação e poder dentre as relações sociais".
} 
consecuentemente, cambiar la realidad a la cual ella está sometida. Obviamente, en este sentido, su discurso también va dirigido a los hombres, pues "A medida que el progreso mejora la condición moral del hombre, él mismo siente la necesidad de elevar a su altura a aquella que Dios le ha dado como compañera"23 (JS, n. 2, 1852, p. 12).

En este aspecto, y con base en las palabras de Manso en el ensayo sobre la "Emancipación moral de la Mujer", que empieza con la declaración de que no es su intención levantar el estandarte de la rebelión, surge la pregunta que ella misma se hace, ¿Qué viene a ser entonces esa tal emancipación moral de la mujer? Para la periodista “es el verdadero conocimiento de la misión de la mujer en la sociedad; es el justo disfrute de sus derechos, de lo que el brutal egoísmo del hombre le roba, y de los cuales la deshereda"24 (JS, n. 2, 1852, p. 12), [la bastardilla es de la autora]. Una mujer emancipada participa del desarrollo del país y en su construcción, caso contrario "¡de qué sirve ilustrar el espíritu de la mujer y abandonarla bajo los cimientos del progreso!"25 (JS, n. 2, 1852, p. 12).

$\mathrm{Su}$ discurso penetra el interior de los hogares, la vida privada, y revela la opresión de la cual las mujeres son víctimas y ve la necesidad de cambio en esa relación: "La mujer conoce la injusticia con la que es tratada y reconoce perfectamente la tiranía del hombre" ${ }^{26}$ (JS, n. 2, 1852, p. 12). Para romper con este círculo vicioso Manso incita a las madres a educar a sus hijos pues, "Mientras la educación del hombre no sea reformada, mientras él considere a la mujer como su propiedad, no habremos hecho nada"27 (JS, n. 2, 1852, p. 12). Además añade otros argumentos para hacerse más convincente: "Todas las madres deben desarraigar muy seriamente este desastroso prejuicio del espíritu de sus hijos; esta idea de superioridad injusta debe desaparecer en el hombre desde niño, porque le es fatal para sí mismo"28 (JS, n. 2, 1852, p. 13).

\footnotetext{
${ }^{23}$ En el original: "à medida que o progresso melhora a condição moral do homem, este mesmo sente a necessidade de elevar a sua altura aquella que Deos the deu por companheira".

${ }^{24}$ En el original: "é o conhecimento verdadeiro da missão da mulher na sociedade; é o justo gozo dos seus direitos, que o brutal egoísmo do homem lhe rouba, e dos quais a desherda".

${ }^{25}$ En el original: "de que serve ilustrar o espírito da mulher, e desampara-lo sob as bases do progresso!".

${ }^{26}$ En el original: "a mulher conhece a injustiça com que é tratada e reconhece perfeitamente a tirania do homem".

${ }^{27}$ En el original: "em quanto a educação do homem se não reformar, em quanto ele considerar a mulher como sua propriedade, nada teremos feito".

${ }^{28}$ En el original: "todas as mães devem mui seriamente desarraigar esse preconceito funesto do espirito de seus filhos; essa idéa de uma superioridade injusta deve desaparecer no homem, desde menino, porque é lhe fatal a elle mesmo".
} 
Al analizar el discurso de Manso en el Jornal das Senhoras, Flores (2014) sostiene que, aunque el discurso de Manso haya producido una diferencia significativa en la discursividad de la época, eso no llegó a provocar una ruptura en el modo de enunciar con relación a los otros periódicos del mismo período. Como afirma dicha autora, "Al proponer una nueva posición para la mujer en la sociedad, ella mantiene a la mujer en la misma posición y formación discursiva (...) que es la de la religión y la

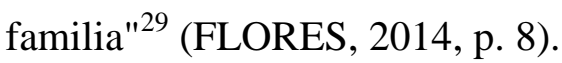

Sin embargo entendemos que Manso, por su coraje, y quizá por su experiencia personal de esposa y madre, revela en su periódico intimidades y verdades con relación al amor conyugal que nos hace observar que una semilla de clamor por respeto y dignidad haya sido plantada ahí. Cuando la periodista afirma que el amor no sobrevive en una relación de esclavitud, puesto que, "ante la superioridad de uno de los sexos -el amor- se marchita, desaparece, y cambia su dulce risa en lágrimas silenciosas" ${ }^{30}$ (JS, n. 2, 1852, p. 13); cuando la periodista se muestra indignada y revela la tiranía insoportable que la mujer encuentra en el casamiento, al ser vista por el marido como un objeto cualquiera al referirse a ella diciendo: "-Mi esposa- con la misma entonación de voz con que dice -mi caballo, mis botas, etc., etc." ${ }^{31}$ (JS, n. 2, 1852, p. 13); cuando la periodista cuestiona el modelo patriarcal de comportamiento sumiso impuesto a la mujer, que la critica si mira, si habla, si sonríe: “¿Podrá una mujer no ser modesta sino siempre mirando al suelo y respondiendo por monosílabos? ¿Acaso la virtud es similar al autoritarismo?"32 (JS, n. 2, 1852, p. 13).

Todo este diálogo entablado entre la redactora y las lectoras, que son el blanco del discurso, nos muestra que Manso, al proponer la emancipación moral de las mujeres -y para ello reivindicaba el derecho a la educación-, enseñó, además, a esas mismas mujeres la necesidad de un cambio en su comportamiento, lo que hoy se suele llamar “empoderamiento femenino". La educación y la ilustración sin un cambio de actitud

\footnotetext{
${ }^{29}$ En el original: "ao propor uma nova posição para a mulher na sociedade, ela mantém a mulher na mesma posição e formação discursiva (...) que é a da religião e da família”.

${ }^{30}$ En el original: "ante a superioridade de um dos sexos - o amor - se definha, desaparece, e troca o seu fagueiro riso em lagrimas silenciosas".

${ }^{31}$ En el original: "- Minha mulher - com a mesma entonação de voz com que diz - meu cavalo, minhas botas, etc., etc.".

${ }^{32}$ En el original: "não poderá uma senhora ser modesta senão olhando sempre para o chão, e respondendo por monosyllabos? A virtude semelha-se por ventura ao autoritarismo?”.
} 
frente al mundo que se nos impone son como un fruto que se pudre en el árbol y no es cosechado.

\section{Consideraciones finales}

Al pensar en el título para este estudio definimos el Jornal das Senhoras como un proyecto periodístico para la emancipación de las mujeres brasileñas, porque creemos que así Manso lo hubiera pensado. No solo por la característica de ese tipo de publicación, con páginas continuas, publicación de folletín y/u otras materias que tienen continuidad en los números siguientes, etc., sino también porque la periodista aporta continuamente una posición la cual no se entiende como un "veredicto", sino como un diálogo que conduce progresivamente a persuadir a las lectoras a aceptar su punto de vista e identificarse con la ideología del Jornal (LUKÁCS apud LANDRUS, 2011, p. 719). Manso se preocupa con el destino de sus palabras, de no estar predicando en el desierto, por eso pide a sus lectoras para "atar este Periódico bien encuadernadito",33 (JS, n. 2, 11 enero 1852, p. 12), para que sus palabras no se conviertan en papel para empaquetar mercancía.

Cabe añadir que el proyecto idealizado por Juana Manso y compartido con las redactoras Violante y Gervasia, que dieron continuidad al periódico, fue innovador al reconocer la condición de sumisión de la mujer en una sociedad patriarcal y al dar visibilidad a estas cuestiones en las páginas del Jornal, además de intentar superarlas.

Como afirma Barbosa (2016, p. 7), el Jornal das Senhoras fue utilizado como objeto de diálogo, instrucción y poder, constituyendo el primer paso para que otras mujeres ascendieran socialmente a la vida profesional.

\footnotetext{
${ }^{33}$ En el original: "encadernar este Jornal, bem encadernadinho".
} 


\section{REFERENCIAS}

ANTELO, Raúl. O jornal da senhora de Noronha. Cuadernos de Literatura, v. XX, n. 39, p. 201-228, ene./jun, 2016. Disponible en:

<https://periodicos.ufsc.br/index.php/nelic/article/view/28426>. Acceso el: 02 ago 2019.

BARBOSA, Everton Vieira. A emancipação feminina no Brasil Oitocentista (18521855). In: ENCONTRO DE HISTÓRIA DA ANPUH-RIO, 17, 2016, Rio de Janeiro. Anais [...]. Rio de Janeiro: Anpuh-Rio/UFRRJ, 2016. Disponible en: <http://www.encontro2016.rj.anpuh.org/resources/anais/42/1466909001_ARQUIVO_ar tigo_final.pdf>. Acceso el: 30 jul. 2019.

BARBOSA, Everton Vieira. Mapeando as tipografias do periódico O Jornal das Senhoras (1852-1855): as relações sócio-espaciais nos impressos, (s,f), p. 1-17. Disponible en: <http://www.seo.org.br/images/Everton_Barbosa.pdf>. Acceso el: 30 jul. 2019.

BATTICUORE, Graciela. La mujer romántica. Lectoras, autoras y escritores en la Argentina: 1830-1870. Buenos Aires: Edhasa, 2005.

BEAUVOIR, Simone. O segundo sexo: 1 fatos e mitos. Trad. Sérgio Milliet. Rio de Janeiro: Nova Fronteira, 2002.

COSTA, Carlos. A revista no Brasil do século XIX. A história da formação das publicações, do leitor e da identidade do brasileiro. São Paulo: Alameda, 2012.

FLORES, Giovanna G. Benedetto. O Jornal das Senhoras e a subjetivação do feminino no jornal dedicado as mulheres. Alcar Sul, Santa Catarina, p. 1-11, 2014. Disponible en: $<$ http://alcarsul2014.sites.ufsc.br/wp-content/uploads/2014/10/gthistoriografiadamidiaGiovanna-B-Flores.dc-1.pdf>. Acceso el: 18 jul. 2018.

HEREDIA, José María. Plan de Estudios. Poesías líricas. Paris: G. Hermanos, 1893. 
LAJOLO, Marisa; ZILBERMAN, Regina. A formação da leitura no Brasil. São Paulo: Unesp, 2019.

LANDRUS, Vanessa. Mujeres al mando de la imprenta: la educación científica de la mujer en la prensa femenina argentina del siglo XIX. Revista Iberoamericana, Pittsburgh, v. LXXVII, n. 236-237, p. 717-730, jul./dic, 2011. Disponible en: <http://revista-iberoamericana.pitt.edu/ojs/index.php/Iberoamericana/article/ view/6850/7015>. Acceso el: 24 set 2018.

LERNER, Gerda. La creación del patriarcado. Trad. Mónica Tusell. Barcelona: Editorial Crítica, 1990.

MANSO, Juana Paula. O Jornal das Senhora, 1852. Disponible en: <http://hemerotecadigital.bn.br/acervo-digital/jornal-senhoras/700096>. Acceso el: 10 fev 2017.

MUZART, Zahidé Lupinacci. Uma espiada na imprensa das mulheres no século XIX. Revista de Estudos Feministas, Florianópolis, v. 11, n. 1, p. 225-233, jan./jun, 2003. Disponible en: <https://doi.org/10.1590/S0104-026X2003000100013>. Acceso el: 22 jul 2019.

PERLIN, Rafaela Luvison; KISTMANN, Virginia Borges. A percepção da moda sem gênero na visão do público. Estudos em design, Rio de Janeiro, v. 26, n. 1, p. 5-28, 2018. Disponible en:

$<$ https://estudosemdesign.emnuvens.com.br/design/article/download/5/287>. Acceso el: 22 jul 2019.

PERROT, Michelle. Minha história das mulheres. Trad. Angela M. S. Corrêa. São Paulo: Contexto, 2017.

PRATT, Mary Louise. Las mujeres y el imaginario nacional en el siglo XIX. Revista de Crítica Literaria Latinoamericana, Lima, año 19, n. 38, p. 51-62, 2do sem, 1993. 
VICENS, María. Entretenimiento, público y autoría femenina en los periódicos de Juana Manso (1850-1860). Perífrasis - Revista Literaria Teoría Crítica, Bogotá, v. 8, n. 16, p. 48-63, jul./dic., 2017. Disponible en:

<http://www.scielo.org.co/pdf/peri/v8n16/v8n16a04.pdf>. Acceso el: 15 jul 2019.

ZUCCOTTI, Liliana. Gorriti, Manso: de las Veladas literarias a "Las conferencias de maestra". In: FLETCHER, Lea. (Comp.) Mujeres y cultura en la Argentina del siglo XIX. Buenos aires: Feminaria, 1994, p. 96-107. 